\title{
Comparison of perioperative outcomes in pancreatic head cancer patients following either a laparoscopic or open pancreaticoduodenectomy with a superior mesenteric artery first approach
}

\author{
Min Young Park*, Woohyung Lee*, Jaewoo Kwon, Ki Byung Song, Dae Wook Hwang, Jae Hoon Lee, Song Cheol Kim \\ Division of Hepato-Biliary and Pancreatic Surgery, Department of Surgery, \\ Asan Medical Center, University of Ulsan College of Medicine, Seoul, Korea
}

Backgrounds/Aims: A superior mesenteric artery first approach (SFA) technique can improve the complete resection rate. It can be used to determine whether an operation can be performed by invading the superior mesenteric artery before performing a pancreatic transection in patients with pancreatic ductal adenocarcinoma (PDAC). The aim of this study was to compare perioperative outcomes between laparoscopic and open SFA for PDAC.

Methods: Between January 2017 and August 2019, consecutive patients who underwent laparoscopic and open pancreaticoduodenectomy (PD) for PDAC using SFA procedures were included and compared between laparoscopic and open procedures.

Results: Fourteen and 83 patients underwent laparoscopic and open surgeries, respectively. In perioperative outcomes, there were no significant differences in the amount of intraoperative blood loss or transfusion rate between the two groups. In the laparoscopic group, the operation time was longer with less patients showing wound infection. R0 resection rate and the number of retrieved lymph nodes showed no significant difference. The average time to adjuvant chemotherapy was longer in the open group. There was no significant difference in the mean survival time or the recurrence free period.

Conclusions: Patients who underwent laparoscopic PD using SFA showed perioperative outcomes comparable compared to those of patients who underwent open procedures performed by experienced surgeons.

Key Words: Carcinoma, pancreatic ductal; Pancreaticoduodenectomy; Mesenteric artery, superior; Laparoscopes

\section{Received: October 22, 2020, Revised: February 24, 2021,}

Accepted: March 2, 2021

\section{Co-Corresponding author: Song Cheol Kim} Division of Hepato-Biliary and Pancreatic Surgery, Department of Surgery, Asan Medical Center, University of Ulsan College of Medicine, 88 Olympic-ro 43-gil, Songpa-gu, Seoul 05505, Korea

Tel: +82-2-3010-3936, Fax: +82-2-474-9027, E-mail: drksc@amc.seoul.kr ORCID: https://orcid.org/0000-0003-4552-4169

Co-Corresponding author: Jae Hoon Lee

Division of Hepato-Biliary and Pancreatic Surgery, Department of Surgery, Asan Medical Center, University of Ulsan College of Medicine, 88 Olympic-ro 43-gil, Songpa-gu, Seoul 05505, Korea

Tel: +82-2-3010-1521, Fax: +82-2-474-9027, E-mail: gooddr23@naver.com ORCID: https://orcid.org/0000-0002-6170-8729

*These authors contributed equally to this study.

Copyright (C) The Korean Association of Hepato-Biliary-Pancreatic Surgery This is an Open Access article distributed under the terms of the Creative Commons Attribution Non-Commercial License (http://creativecommons.org/licenses/by-nc/4.0) which non-commercial use, distribution, and reproduction in any medium, provided the original permits unrestricted work is properly cited.

\section{INTRODUCTION}

Pancreatic ductal adenocarcinoma (PDAC) is known as one of high mortality cancers [1,2], accounting for about $85 \%$ of all pancreatic cancers [2]. To date, PDAC has a 5 -year survival rate of less than $5 \%[2,3]$. Its prevalence is increasing due to an aging society $[3,4]$. Genetic, diabetes, obesity, and smoking are known as causes of pancreatic cancer $[1,5,6]$. There are no useful screening tests known for pancreatic cancer or recommended [7]. Therefore, the stage is often advanced at the time of diagnosis, which affects the prognosis of PDAC [2].

Surgery is the only known treatment to cure PDAC. Additional adjuvant chemotherapy can improve survival [1]. In PDAC, minimally invasive surgery (MIS) can reduce the number of hospital days with a benefit for pain reduction. In distal pancreatectomy, MIS shows similar results to open surgery. In 
pancreaticoduodenectomy (PD), many studies have revealed the safety of MIS $[8,9]$.

It is known that tumor invasion at resection margin affects the prognosis after surgical resection. As a result, many efforts have been made to improve surgical outcomes and improve the complete resection rate with the development of surgical techniques and equipment [10-12]. In this context, the superior mesenteric artery first approach (SFA) technique has emerged. Conventional PD first ligates the inferior pancreaticoduodenal artery (IPDA) before pancreatic resection. Blocking the blood flow can reduce the amount of bleeding and prevent damage to nearby blood vessels such as the right hepatic artery (RHA) from superior mesenteric artery (SMA). Complete removal of the surrounding soft tissue can obtain a sufficient number of lymph nodes and increase the rate of complete resection of the tumor [13-15].

With the development of surgical methods, there has been an intermediate classification between resectable pancreatic cancer and locally advanced pancreatic cancer (LAPC) that cannot be surgically resected. It is called borderline resectable pancreatic cancer (BRPC) $[16,17]$. The definition of BRPC means when the tumor covers part of the hepatic artery or it is attached to the SMA, the superior mesenteric vein (SMV) or part of the portal vein is narrowed by the adjacent tumor $[16,18]$. In this case, surgery may be possible by reconstructing some of the vessels after a combined resection. However, it can increase the incidence of incomplete resection and postoperative complications $[19,20]$. The most common invasion of the tumor, which was confirmed by postoperative specimens, was the cutsurface to the SMA [13]. To overcome these limitations, the SFA was introduced. Patients with advanced pancreatic cancer are unsuitable to undergo surgical resection if there is a tumor invasion in the adventitia of the SMA during surgery. In fact, the presence of vascular invasion can only be determined intraoperatively. The SFA can be used to determine the invasion of the SMA before pancreatic resection and to decide if surgery is possible [15].

The SFA technique is widely used in open surgery. Various studies have shown that surgical complications of SFA do not differ from those of a conventional procedure [13,21]. It can also bring about complete resection rate, increase survival rate [22], and reduce bleeding amount [23]. However, research about the safety of SFA technique in laparoscopic procedures is limited. Thus, the purpose of this study was to determine whether a surgery using the SFA technique in laparoscopic PD would differ in safety and prognosis compared to an open surgery.

\section{MATERIALS AND METHODS}

Between January 2017 and August 2019, patients at a tertiary referral center who underwent laparoscopic and open $\mathrm{PD}$ using SFA procedures for PDAC were analyzed in this study.
Surgical techniques were reviewed through recorded video for patients undergoing laparoscopic surgery. Patients included in this study were those considered to have resectable PDAC. Patients with clinically stage T4, concurrent distant metastasis, concurrent malignancy, and prior history of malignancy were excluded from this study.

Preoperative imaging was performed to determine the feasibility of surgery based on the criteria for BRPC and LAPC following the National Comprehensive Cancer Network (NCCN) guideline [17]. The SFA technique was used for the resection after confirming that there was no tumor invasion at the SMA. Complete resection of the tumor was possible by hanging the SMA first. It can be divided into three levels according to the range of soft tissue dissection around the pancreas. The first level simply refers to the separation of soft tissues around the pancreas from blood vessels without lymph node dissection. The second level is a method of ligation of the IPDA at the baseline to remove the surrounding lymph nodes in en-bloc while preserving nerve bundles around the SMA. The third level is a method of obtaining complete resection of the tumor from perineural spread by removing both soft tissues and nerve bundles located $180^{\circ}$ to the right side of the SMA $[15,24]$. In this study, the level 3 method was used to remove all soft tissues and nerves on the right side of the SMA (Fig. 1; Supplementary Video 1). Data about the characteristics of patients (age, sex, American Society of Anesthesiologists (ASA) score [25], neoadjuvant chemotherapy, body mass index), preoperative laboratory findings (blood carcinoembryonic antigen [CEA], carbohydrate antigen 19-9 [CA19-9], total bilirubin), preoperative patient performance, blood loss during surgery, operation time, postoperative complications (pancreatic fistula, bile leakage, diarrhea, delayed gastric emptying, bleeding, surgical wound infection), and oncologic outcome were collected. Based on the collected data, results were compared between the

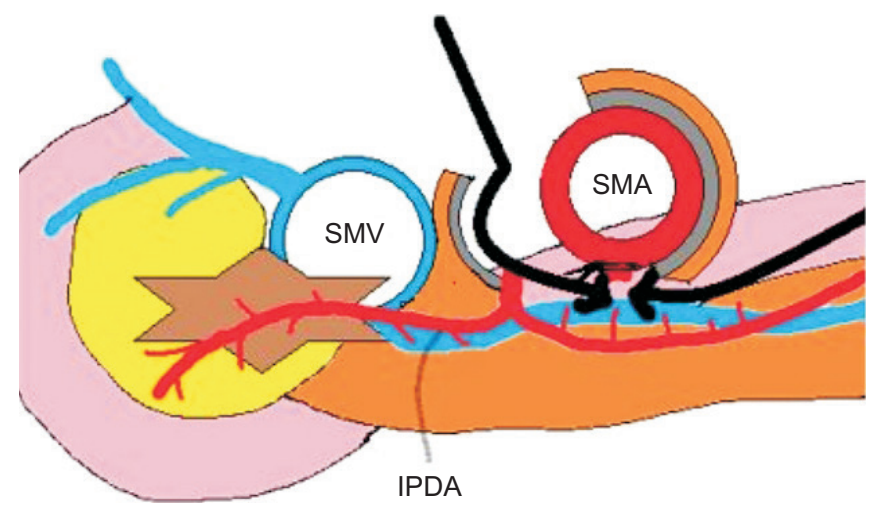

Fig. 1. The range of soft tissue dissection around the pancreas with the SMA first approach technique. Surgical extents included all soft tissues and nerve bundles located $180^{\circ}$ to the right side of the SMA. SMV, superior mesenteric vein; SMA, superior mesenteric artery; IPDA, inferior pancreaticoduodenal artery. 
laparoscopic group and the open SFA group. This study was approved by the Institutional Review Board of Asan Medical Center (No. 2020-1330).

\section{Surgical procedures}

After abdominal access was established, the greater omentum was divided using an energy device and the right colon was separated and fully mobilized from the liver and duodenum. The retropancreatic SMV was then exposed and the right gastroepiploic vessels were transected. After removing soft tissues from around the SMV and the SMA, each was hung with a vessel loop. Mobilization of the duodenum to the Treitz ligament was performed with traction of the duodenum by a surgical assistant. The stomach and the duodenum were divided using a linear stapler.

After cholecystectomy, dissection of the hepatoduodenal ligament and isolation of the common bile duct were performed. The right and left hepatic arteries were identified and isolated. Lymph node dissection was then performed. The gastrohepatic ligament was opened to visualize the superior border of the pancreas and to identify the common hepatic artery. The right gastric artery and the gastroduodenal artery were identified and transected using a Hem-o-lock clip. The pancreas was divided above the SMV using an energy device. After retracting the resected pancreas to the right side of the patient's abdomen, the portal vein was identified and hung with a vessel loop. The jejunum was divided approximately $10-15 \mathrm{~cm}$ distal to the Treitz ligament with a linear stapler. An energy device and an electrocautery were used to separate the remaining soft tissues and branches from the SMA between the uncinate process of the pancreas and the SMA to complete the resection. Pancreatojejunostomy was performed using a double-layered, endto-side, duct-to-mucosa method. A polyethylene internal stent was inserted into the pancreatic duct. End-to-side hepaticojejunostomy was performed using continuous suturing at the posterior wall with interrupted or continuous suturing at the anterior wall. Duodenojejunostomy or gastrojejunostomy with jejunojejunostomy was performed. Two or three closed suction drains were placed at the superior and inferior borders of the pancreatojejunostomy site.

\section{Statistical analysis}

For statistical analysis, chi-square test was used for univariate analysis of discontinuity variables and Student's t-test was used for univariate analysis of continuity variables. Both methods were interpreted as statistically significant results when $p$-value was less than 0.05 . Multivariate analysis was performed for variables with $p$-value $<0.1$ in univariate analysis, including MIS. The odds ratios (ORs) and 95\% confidence intervals (CIs) were identified using a logistic regression model. The overall survival and disease-free survival were analyzed using the Kaplan-Meier technique. All statistical analyses were performed using IBM SPSS version 26.0 (IBM Corp., Armonk, NY, USA).

\section{RESULTS}

\section{Demographics}

There were 14 patients in the laparoscopic group and 83 patients in the open group. The median age of the two groups was same as 65 years old $(p=0.493)$. The median body mass index (BMI) was $21.5 \mathrm{~kg} / \mathrm{m}^{2}\left(17.5-24.0 \mathrm{~kg} / \mathrm{m}^{2}\right)$ in the laparoscopic group and $22.5 \mathrm{~kg} / \mathrm{m}^{2}\left(17.5-29.3 \mathrm{~kg} / \mathrm{m}^{2}\right)$ in the open group $(p$ $=0.025)$. Preoperatively identified ASA classification system categories belonged to category II in both groups, showing no significant difference $(92.9 \%$ vs. $94.0 \%, p=0.136)$. According to the blood test for preoperative tumor markers, CEA showed a median of $2.0 \mathrm{ng} / \mathrm{mL}$ in the laparoscopic group and a median of $3.4 \mathrm{ng} / \mathrm{mL}$ in the open group $(p=0.198)$. CA19-9 was not significantly different between the two groups either, with a median of $48.7 \mathrm{U} / \mathrm{mL}$ in the laparoscopic group and a median

Table 1. Demographics of subjects analyzed in this study

\begin{tabular}{lccc}
\hline \multicolumn{1}{c}{ Factor } & Laparoscopic group $(\mathrm{n}=14)$ & Open group $(\mathrm{n}=83)$ & $p$-value \\
\hline Sex (female $:$ male) & $7: 7$ & $40: 43$ & 0.902 \\
Age $(\mathrm{yr})$ & $65(36-78)$ & $65(40-87)$ & 0.493 \\
BMI $\left(\mathrm{kg} / \mathrm{m}^{2}\right)$ & $21.5(17.5-24.0)$ & $22.5(17.5-29.3)$ & 0.025 \\
ASA score & $2(2-2)$ & $2(2-2)$ & 0.136 \\
CEA $(\mathrm{ng} / \mathrm{mL})$ & $2.0(1.2-5.8)$ & $3.4(0.35-37.6)$ & 0.198 \\
CA19-9 $(\mathrm{U} / \mathrm{mL})$ & $48.7(11.6-575.0)$ & $72.8(0.6-10422.0)$ & 0.376 \\
Total bilirubin $(\mathrm{mg} / \mathrm{dL})$ & $5.1(0.2-5.3)$ & $0.6(0.1-8.3)$ & 0.851 \\
Neoadjuvant CTx & $1(7.1)$ & $16(19.3)$ & 0.643 \\
Resectability & & & 0.309 \\
Resectable & $9(64.3)$ & $64(77.1)$ & $19(22.9)$ \\
Borderline & $5(35.7)$ & &
\end{tabular}

Values are presented as number only, mean (range), or number (\%).

BMI, body mass index; ASA, American society of anesthesiologists; CEA, carcinoembryonic antigen; CA19-9, carbohydrate antigen 19-9; CTx, chemotherapy. 
of $72.8 \mathrm{U} / \mathrm{mL}$ in the open group ( $p=0.376)$. According to the NCCN classification criteria confirmed by preoperative imaging, 9 patients $(64.3 \%)$ were resectable in the laparoscopic group and 5 patients $(35.7 \%)$ were borderline resectable. In the open group, 64 patients $(77.1 \%)$ were resectable and 19 patients (22.9\%) were borderline resectable. One patient $(7.1 \%)$ in the laparoscopic group and 16 patients (19.3\%) in the open group underwent neoadjuvant chemotherapy, showing no significant difference between the two groups ( $p=0.309$; Table 1).

\section{Intra-operative outcomes}

There was no significant differences in blood loss $(340 \mathrm{~mL}$ vs. $386 \mathrm{~mL} ; p=0.688)$ or transfusion rate $(7.1 \%$ vs. $14.5 \% ; p=0.526)$ between laparoscopic and open groups. The total operation time was significantly longer in the laparoscopic group (422 vs. 336 minutes; $p<0.001$ ). However, the number of cases requiring combined resection of portal vein showed no statistically significant difference (7.1\% vs. 9.6\%; $p=0.769$; Table 2).

\section{Postoperative complications}

Complication rate was not significantly different (28.6\% vs. $38.6 \% ; p=0.480)$. There was no significant difference in severe complication of Clavien-Dindo classification 3 or more between the two groups ( 1 vs. $0, p=0.684$ ). There was no patient $(0 \%)$ with wound infection in the laparoscopic group whereas there were 8 patients $(9.6 \%)$ in the open surgery group ( $p=$ $0.004)$. Pancreatic fistulas were found in 2 patients $(14.3 \%)$ in the laparoscopic group and 18 patients $(21.6 \%)$ in the open group. Bile leakage was absent in both groups. Bleeding was noted in 3 patients $(3.6 \%)$ in the open group. Of these three patients, two were found to have intraperitoneal hematoma on regular computed tomography (CT) follow-up after surgery without requiring an additional treatment such as intervention. Only one patient needed intensive care because of volume overload following respiratory failure. Delayed gastric emptying was found in 4 patients (4.8\%) in the open group that required conservative treatment ( 4 vs. $0 ; p=0.407$ ).

Symptoms of diarrhea that required medication were found in 4 patients $(28.6 \%)$ in the laparoscopic group and 9 patients $(10.8 \%)$ in the open group. None of these patients died within 90 days after surgery. One patients (7.1\%) in the laparoscopic group and five patients $(6.0 \%)$ in the open group were required to be re-admitted within 60 days. The mean hospital stay was 10 days in the laparoscopic group and 12.7 days in the open group $(p=0.319)$.

Postoperative adjuvant chemotherapy in our center was performed for 12 patients (85.7\%) in the laparoscopic group and 61 patients $(73.5 \%)$ in the open group. The average time to postoperative adjuvant chemotherapy in the laparoscopic group was shorter than that in the open group (36.1 vs. 53.6 days; $p=$

Table 2. Intraoperative and postoperative outcomes

\begin{tabular}{|c|c|c|c|}
\hline Factor & Laparoscopic group $(n=14)$ & Open group $(n=83)$ & $p$-value \\
\hline $\mathrm{EBL}(\mathrm{mL})$ & $340(0-990)$ & $386(0-2,265)$ & 0.688 \\
\hline Transfusion & $1(7.1)$ & $12(14.5)$ & 0.526 \\
\hline OP time (min) & $422(371-487)$ & $336(211-558)$ & $<0.001$ \\
\hline PVR & $1(7.1)$ & $8(9.6)$ & 0.769 \\
\hline Overall complications & $4(28.6)$ & $32(38.6)$ & 0.480 \\
\hline Severe complications (Clavien-Dindo $\geq 3$ ) & $0(0)$ & $1(1.2)$ & 0.684 \\
\hline POPF & & & 0.532 \\
\hline$B L$ & $2(14.3)$ & $16(19.2)$ & \\
\hline Grade B \& C & $0(0)$ & $2(2.4)$ & \\
\hline Bile leakage & $0(0)$ & $0(0)$ & - \\
\hline Bleeding & $0(0)$ & $3(3.6)$ & 0.475 \\
\hline DGE & $0(0)$ & $4(4.8)$ & 0.407 \\
\hline Diarrhea & $4(28.6)$ & $9(10.8)$ & 0.192 \\
\hline Wound infection & $0(0)$ & $8(9.6)$ & 0.004 \\
\hline Hospital stay (day) & $10(7-16)$ & $12.7(7-96)$ & 0.319 \\
\hline Mortality within 90 days & $0(0)$ & $0(0)$ & - \\
\hline Readmission within 60 days & $1(7.1)$ & $5(6.0)$ & 0.885 \\
\hline \multicolumn{4}{|l|}{ Adjuvant CTx } \\
\hline Patients number & $12(85.7)$ & $61(73.5)$ & 0.871 \\
\hline Days until CTx (days) & 36.1 & 53.6 & 0.016 \\
\hline Additional IV pain killer & 11.6 & 12.4 & 0.806 \\
\hline
\end{tabular}

Values are presented as mean (range) or number (\%).

EBL, estimated blood loss; OP, operation; PVR, portal vein resection; POPF, postoperative pancreatic fistula; BL, biochemical leakage; DGE, delayed gastric emptying; CTx, chemotherapy; IV, intravenous. 
Table 3. Factors affecting postoperative complications

\begin{tabular}{|c|c|c|c|c|c|c|c|c|}
\hline \multirow{3}{*}{ Factor } & \multicolumn{4}{|c|}{ Univariate } & \multicolumn{4}{|c|}{ Multivariate } \\
\hline & \multirow{2}{*}{ OR } & \multicolumn{2}{|c|}{$95 \% \mathrm{Cl}$} & \multirow{2}{*}{$p$-value } & \multirow{2}{*}{ OR } & \multicolumn{2}{|c|}{$95 \% \mathrm{Cl}$} & \multirow{2}{*}{$p$-value } \\
\hline & & LB & UB & & & LB & UB & \\
\hline MIS & 0.638 & 0.181 & 2.241 & 0.479 & 0.857 & 0.227 & 3.242 & 0.819 \\
\hline Age $(y r)$ & 1.054 & 1.003 & 1.106 & 0.036 & 1.054 & 1.002 & 1.109 & 0.041 \\
\hline Sex & 1.292 & 0.559 & 2.986 & 0.546 & & & & \\
\hline BMI $\left(\mathrm{kg} / \mathrm{m}^{2}\right)$ & 1.164 & 0.990 & 1.368 & 0.065 & 1.160 & 0.978 & 1.376 & 0.088 \\
\hline Log. CA19-9 & 0.936 & 0.768 & 1.141 & 0.509 & & & & \\
\hline NCCN resectability & 0.616 & 0.238 & 1.591 & 0.313 & & & & \\
\hline Neoadjuvant CTx & 2.298 & 0.751 & 7.032 & 0.140 & & & & \\
\hline Log. Total bilirubin & 0.927 & 0.591 & 1.455 & 0.740 & & & & \\
\hline ASA 2 & 0.569 & 0.075 & 4.344 & 0.583 & & & & \\
\hline ASA 3 & 1.000 & 0.032 & 31.186 & 1.000 & & & & \\
\hline
\end{tabular}

OR, odd ratios; $\mathrm{Cl}$, confidence interval; LB, lower bound; UB, upper bound; MIS, minimally Invasive Surgery; BMI, body mass index; CA19-9, carbohydrate antigen 19-9; NCCN, National Comprehensive Cancer Network; CTX, chemotherapy; ASA, American Society of Anesthesiologists.

$0.016)$.

After surgery, all patients took intravenous patient-controlled analgesia and per os analgesics. The average number of additional IV analgesics was 11 times in the laparoscopic group and 12 times in the open group without statistical significance ( $p$ $=0.806$; Table 2). Univariate analysis revealed factors affecting postoperative complications. Resectability according to NCCN guideline of CT image, MIS, neoadjuvant chemotherapy, sex, body mass index, CA19-9, and serum bilirubin levels did not significantly affect postoperative complications. Only the correlation between age and complication rate was significant (OR, 1.054; 95\% CI, 1.003-1.106; $p=0.036$ ). Multivariate analysis revealed that whether MIS was done (OR, 0.638; 95\% CI, $0.181-2.241 ; p=0.479)$, age (OR, $1.054 ; 95 \% \mathrm{CI}, 1.003-1.106 ; p$ $=0.036)$, and BMI (OR, 1.164; 95\% CI, 0.990-1.368; $p=0.065)$ were significant variables affecting postoperative complications. MIS did not affect complications (OR, 0.857; 95\% CI, $0.227-3.242 ; p=0.819)$. BMI was not a significant variable affecting postoperative complications either (OR, 1.160; 95\% CI, $0.978-1.376 ; p=0.088$ ). Only age showed a statistically significant association with complication rates (OR, 1.054; 95\% CI, 1.002-1.109; $p=0.041$; Table 3).

\section{Pathologic results and oncologic outcomes}

The average size of tumors after surgery was $2.3 \mathrm{~cm}$ in the laparoscopic group and $2.8 \mathrm{~cm}$ in the open group $(p=0.274)$. There was no statistically significant difference in cell differentiation. More than $70 \%$ of patients showed moderate differentiation in both groups $(p=0.517)$. Lymphovascular invasion was observed with 9 patients $(64.3 \%)$ in the laparoscopic group and 46 patients $(55.4 \%)$ in the open group $(p=0.604)$. Perineural invasion was also observed with 10 patients $(71.4 \%)$ in the laparoscopic group and 61 patients $(73.5 \%)$ in the open group $(p=0.761)$. Total number of lymph nodes harvested during
Table 4. Pathologic outcomes

\begin{tabular}{lccc}
\hline \multicolumn{1}{c}{ Factor } & $\begin{array}{c}\text { Laparoscopic } \\
\text { group } \\
(\mathrm{n}=14)\end{array}$ & $\begin{array}{c}\text { Open group } \\
(\mathrm{n}=83)\end{array}$ & $p$-value \\
\hline Tumor size $(\mathrm{cm})$ & $2.3(1.5-4.3)$ & $2.8(0-6.3)$ & 0.274 \\
Retrieved LN & $17(9-24)$ & $20(4-61)$ & 0.073 \\
R1 resection margin & $3(21.4)$ & $17(20.5)$ & 0.936 \\
Cell differentiation & & & 0.517 \\
WD & $2(14.3)$ & $13(15.7)$ & \\
MD & $12(85.7)$ & $60(72.3)$ & \\
PD & $0(0)$ & $9(10.8)$ & 0.604 \\
Lymphovascular & $9(64.3)$ & $46(55.4)$ & \\
invasion & $10(71.4)$ & $61(73.5)$ & 0.761 \\
\hline
\end{tabular}

Values are presented as mean (range) or number (\%).

LN, lymph node; WD, well differentiation; MD, moderate differentiation PD, poorly differentiation.

surgery as a factor affecting the survival of patients was 17 in the laparoscopic group and 20 in the open group, showing no significant difference between the two groups $(p=0.073)$. R1 resection rate was $21.4 \%$ in the laparoscopic group and $20.5 \%$ in the open group, showing no significant difference between the two groups ( $p=0.936$; Table 4$)$.

Univariate analysis revealed that $\mathrm{R} 0$ resection was not affected by MIS or tumor resectability determined by preoperative imaging test, neoadjuvant chemotherapy, age, sex, BMI, CA199 , and serum bilirubin levels. In the multivariate analysis, there was no difference in complete resection rate according to the surgical method (OR, 1.180; 95\% CI, 0.283-4.915; $p=0.818$; Table 5).

The median duration of follow-up was 15 months and the median survival time was 18 months. There was no significant 
Table 5. Factors affecting R0 resection

\begin{tabular}{|c|c|c|c|c|c|c|c|c|}
\hline \multirow{3}{*}{ Factor } & \multicolumn{4}{|c|}{ Univariate } & \multicolumn{4}{|c|}{ Multivariate } \\
\hline & \multirow{2}{*}{ OR } & \multicolumn{2}{|c|}{$95 \% \mathrm{Cl}$} & \multirow{2}{*}{$p$-value } & \multirow{2}{*}{ OR } & \multicolumn{2}{|c|}{$95 \% \mathrm{Cl}$} & \multirow{2}{*}{$p$-value } \\
\hline & & LB & UB & & & LB & UB & \\
\hline MIS & 1.059 & 0.261 & 4.301 & 0.936 & 1.180 & 0.283 & 4.915 & 0.818 \\
\hline Age & 0.985 & 0.936 & 1.037 & 0.559 & & & & \\
\hline Sex & 0.925 & 0.341 & 2.507 & 0.877 & & & & \\
\hline BMI & 0.971 & 0.804 & 1.171 & 0.754 & & & & \\
\hline Log. CA19-9 & 1.274 & 0.970 & 1.673 & 0.081 & 1.279 & 0.970 & 1.686 & 0.080 \\
\hline Resectability & 0.712 & 0.235 & 2.153 & 0.543 & & & & \\
\hline Neoadjuvant CTx & 0.663 & 0.168 & 2.624 & 0.555 & & & & \\
\hline Log. Total bilirubin & 1.064 & 0.629 & 1.798 & 0.816 & & & & \\
\hline ASA 2 & 0.230 & 0.029 & 1.796 & 0.159 & & & & \\
\hline ASA 3 & 1.000 & 0.032 & 31.186 & 1.000 & & & & \\
\hline
\end{tabular}

OR, odd ratios; Cl, confidence interval; LB, lower bound; UB, upper bound; MIS: Minimally Invasive Surgery; BMI: Body mass index; CA19-9: Carbohydrate antigen 19-9; CTx: Chemotherapy; ASA: American society of anethesiologists.

A

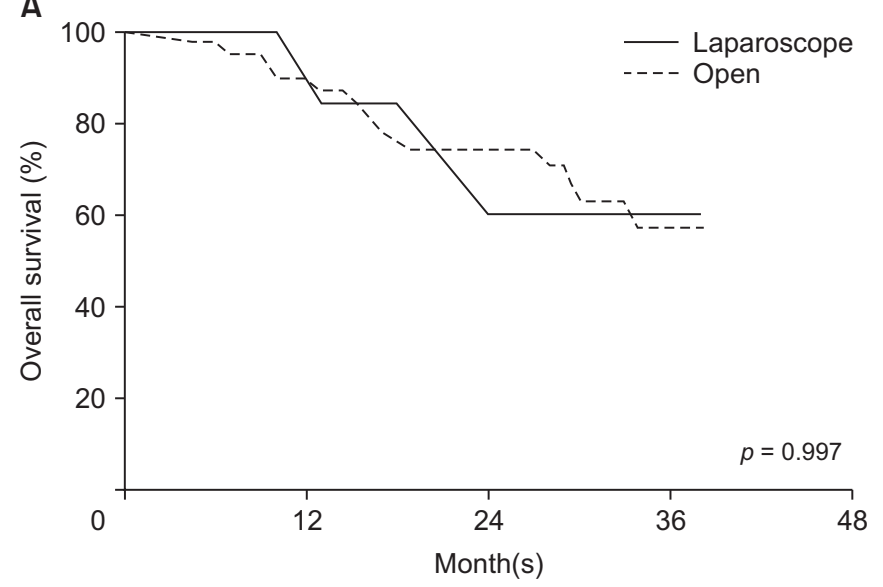

B

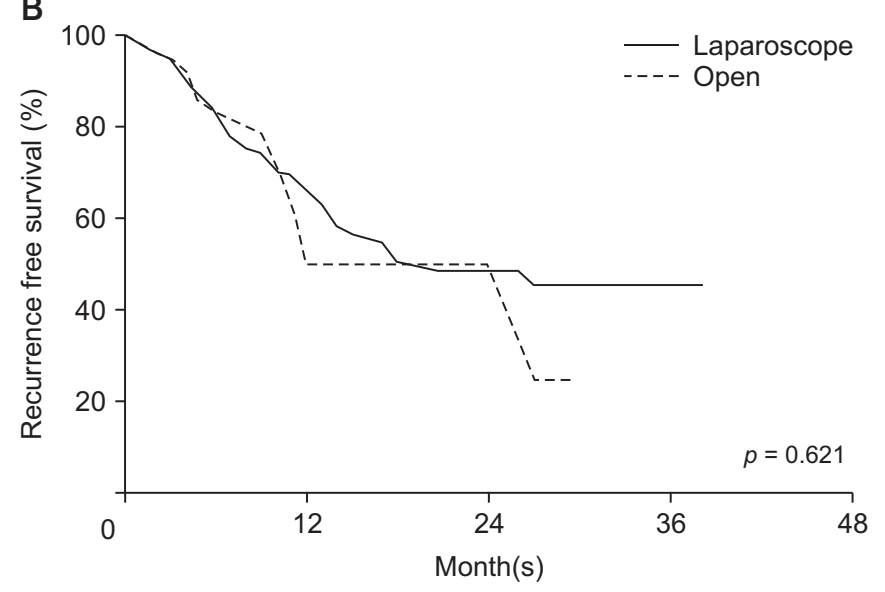

Fig. 2. Kaplan-Meier estimates of overall survival (A) and recurrence free survival (B) for the open SFA group and the laparoscopic SFA group. (A) The mean survival time was 30.8 months $(95 \% \mathrm{Cl}, 25.1-36.5$ months) for the laparoscopic group and 30.5 months (95\% Cl, 27.8-33.2 months) for the open group ( $p=0.997)$. (B) The mean time to recurrence was 18.8 months $(95 \% \mathrm{Cl}, 13.4-24.1$ months) for the laparoscopic group and $23.2 \mathrm{months}(95 \% \mathrm{Cl}$, 19.9-26.6 months) for the open group $(p=0.621)$. SFA, superior mesenteric artery first approach; $\mathrm{Cl}$, confidence interval.

difference in mean survival between the laparoscopic group (30.8 months; 95\% CI, 25.1-36.5 months) and the open group (30.5 months; 95\% CI, 37.8-33.2 month) ( $p=0.997$; Fig. 2).

The median duration of recurrence free survival was 13 months. There was no significant difference in the mean recurrence free survival between the laparoscopic group (18.8 months; 95\% CI, 13.4-24.1 months) and the open group (23.2 months; 95\% CI, 19.9-26.6 months) ( $p=0.621$; Fig. 2). For distant metastasis, about $80 \%$ of them showed hepatic metastasis and the remaining $20 \%$ showed lung metastasis. For patients with recurrent findings, peritoneal seeding was the most common $(40.0 \%)$, followed by recurrence of lymph nodes around the mesenteric artery (22.0\%). Common hepatic artery, inferior vena cava, periaortic lymph nodes, and remaining pancreatic recurrence were also observed.

\section{DISCUSSION}

In resectable pancreatic cancer, SFA technique has the advantage of improving complete resection rate, sufficient lymph node removal, and major peripheral blood vessel damage [1315]. In BRPC or LAPC after neoadjuvant chemotherapy, prior confirmation of SMA invasion can confirm the possibility of surgical resection before dividing the pancreas [22]. Previous studies have shown that the SFA in open surgery can be safely achieved without increasing complications compared to standard procedures [26]. However, a laparoscopic technique with SFA is not well known. This study compared the laparoscopic 
group and the open group of patients who underwent surgery using the SFA.

This study was performed on patients who underwent PD using the SFA technique for PDAC in recent two years. The operation time was significantly longer in the laparoscopic group. However, there was no significant difference in blood loss between the two groups. There was no difference in the incidence of complications between the two groups either after surgery. There were no statistically significant factors affecting the incidence of complications including MIS. However, there was a difference in the time period after surgery and before adjuvant chemotherapy between the two groups. The period from the operation date to adjuvant chemotherapy and the period from discharge date to adjuvant chemotherapy was also longer in the open group ( 46.9 vs. 30.6 days, $p=0.022$ ). This was thought to be because the time to recover the general condition was longer in the open group than in the laparoscopic group. There was no difference in the number of additional IV analgesic medications between the two groups.

The average number of harvested lymph nodes confirmed after surgery and the rate of R0 resection were similar between the two groups. The $\mathrm{R} 0$ resection rate was not affected by MIS. The median duration of follow up was short. However, there was no significant difference in overall survival or recurrence free survival between the two groups.

To date, many studies have shown that laparoscopic PD for the treatment of PDAC can reduce the number of hospital stay compared to open $\mathrm{PD}$, with reduced pain and comparable postoperative complications [27]. Laparoscopic surgery has been recognized as a safe procedure that can be realized as open as a treatment for PDAC $[8,9]$. We analyzed the learning curves of skillful experts in laparoscopic pylorus-preserving pancreaticoduodenectomy. Results showed that after laparoscopic pylorus-preserving pancreaticoduodenectomy for 55 patients with benign disease, the period needed for stabilization and the time required for surgery gradually decreased. After about 100 surgeries, the patient group was expanded to malignant tumors. From about 180 surgical cases, it was confirmed that the operation time was shortened with lymph node dissection in malignant cases and the prognosis was better in complications. In terms of oncological prognosis, these results were similar to those of open surgery [28].

With continuous development of surgical technique, increasing the survival rate by improving $\mathrm{R} 0$ resection has been recognized as an important issue in the treatment of pancreatic head cancer [10-12]. In this context, SFA technique has emerged. The SFA can increase the number of harvested lymph nodes and secure a complete resection margin [13-15]. In addition, it is possible to confirm complete surgical resection for tumors with borderline resectable patients [15]. Currently, many studies have revealed that the SFA can be safely implemented without showing any significant difference in complications or prognosis compared to previous procedures [13,21]. With a laparoscopic procedure, there is a lack of studies. We compared results of laparoscopic SFA with those of open surgery. Postoperative results, complications, and prognosis were not significantly between the two groups. Therefore, SFA can be safely realized by laparoscopy if it is performed by an expert surgeon with sufficient experience in laparoscopic PD.

This study has some limitations. First, it was retrospective in nature. Second, the number of patients included in this study was not sufficient. Due to the small number of patients, propensity score matching could not be performed and the possibility of bias could not be excluded. Third, this study included only patients who underwent PD with SFA. Therefore, we could not compare results between standard PD and SFA. In addition, the average follow up period was 16 months, which was a short-term that could not make a definitive conclusion.

In conclusion, PD using SFA technique can also be considered in a laparoscopic surgery. Perioperative outcomes are comparable between laparoscopic and open SFA groups. Therefore, if practiced by an expert surgeon, SFA is judged to be a safe technique. However, this study has some limitations. A prospective research with more patients is needed in the future.

\section{FUNDING}

This study was supported by a grant (grant number: 2019IT0008-1) from Asan Institute for Life Sciences, Asan Medical Center, Seoul, Korea.

\section{SUPPLEMENTARY DATA}

Supplementary data related to this article can be found at https://doi.org/10.14701/ahbps.2021.25.3.358

\section{CONFLICT OF INTEREST}

No potential conflict of interest relevant to this article was reported.

\section{ORCID}

Min Young Park, https://orcid.org/0000-0002-7444-5075

Woohyung Lee, https://orcid.org/0000-0002-8119-6943

Jaewoo Kwon, https://orcid.org/0000-0001-8922-4374

Ki Byung Song, https://orcid.org/0000-0001-5422-5481

Dae Wook Hwang, https://orcid.org/0000-0002-1749-038X

Jae Hoon Lee, https://orcid.org/0000-0002-6170-8729

Song Cheol Kim, https://orcid.org/0000-0003-4552-4169

\section{AUTHOR CONTRIBUTIONS}

Conceptualization: SCK. Data curation: MYP. Methodology: MYP. Visualization: MYP. Writing - original draft: MYP, WL. Writing - review \& editing: MYP, WL, JK, KBS, DWH, JHL, SCK. 


\section{REFERENCES}

1. McGuigan A, Kelly P, Turkington RC, Jones C, Coleman HG, McCain RS. Pancreatic cancer: a review of clinical diagnosis, epidemiology, treatment and outcomes. World J Gastroenterol 2018;24:4846-4861.

2. Ilic M, Ilic I. Epidemiology of pancreatic cancer. World J Gastroenterol 2016;22:9694-9705.

3. Ferlay J, Soerjomataram I, Dikshit R, Eser S, Mathers C, Rebelo $\mathrm{M}$, et al. Cancer incidence and mortality worldwide: sources, methods and major patterns in GLOBOCAN 2012. Int J Cancer 2015;136:E359-E386.

4. World Health Organization. World health statistics 2015. Geneva: World Health Organization, 2015.

5. Bosetti C, Lucenteforte E, Silverman DT, Petersen G, Bracci PM, Ji BT, et al. Cigarette smoking and pancreatic cancer: an analysis from the International Pancreatic Cancer Case-Control Consortium (Panc4). Ann Oncol 2012;23:1880-1888.

6. Wang YT, Gou YW, Jin WW, Xiao M, Fang HY. Association between alcohol intake and the risk of pancreatic cancer: a dose-response meta-analysis of cohort studies. BMC Cancer 2016;16:212.

7. Shin EJ, Canto MI. Pancreatic cancer screening. Gastroenterol Clin North Am 2012;41:143-157.

8. Correa-Gallego C, Dinkelspiel HE, Sulimanoff I, Fisher S, Viñuela EF, Kingham TP, et al. Minimally-invasive vs open pancreaticoduodenectomy: systematic review and meta-analysis. J Am Coll Surg 2014;218:129-139.

9. de Rooij T, Lu MZ, Steen MW, Gerhards MF, Dijkgraaf MG, Busch OR, et al. Minimally invasive versus open pancreatoduodenectomy: systematic review and meta-analysis of comparative cohort and registry studies. Ann Surg 2016;264:257-267.

10. Demir IE, Jäger C, Schlitter AM, Konukiewitz B, Stecher L, Schorn S, et al. R0 versus R1 resection matters after pancreaticoduodenectomy, and less after distal or total pancreatectomy for pancreatic cancer. Ann Surg 2018;268:1058-1068.

11. Geer RJ, Brennan MF. Prognostic indicators for survival after resection of pancreatic adenocarcinoma. Am J Surg 1993;165:68-72; discussion 72-73.

12. Nitecki SS, Sarr MG, Colby TV, van Heerden JA. Long-term survival after resection for ductal adenocarcinoma of the pancreas. Is it really improving? Ann Surg 1995;221:59-66.

13. Weitz J, Rahbari N, Koch M, Büchler MW. The "artery first" approach for resection of pancreatic head cancer. J Am Coll Surg 2010;210:e1-e4.

14. Sabater L, Cugat E, Serrablo A, Suarez-Artacho G, Diez-Valladares L, Santoyo-Santoyo J, et al. Does the artery-first approach improve the rate of R0 resection in pancreatoduodenectomy?: a multicenter, randomized, controlled trial. Ann Surg 2019;270:738-746.

15. Inoue Y, Saiura A, Oba A, Kawakatsu S, Ono Y, Sato T, et al. Optimal extent of superior mesenteric artery dissection during pancreaticoduodenectomy for pancreatic cancer: balancing surgical and oncologi- cal safety. J Gastrointest Surg 2019;23:1373-1383.

16. Varadhachary GR, Tamm EP, Abbruzzese JL, Xiong HQ, Crane CH, Wang $\mathrm{H}$, et al. Borderline resectable pancreatic cancer: definitions, management, and role of preoperative therapy. Ann Surg Oncol 2006;13:1035-1046.

17. Tempero MA, Malafa MP, Al-Hawary M, Asbun H, Bain A, Behrman SW, et al. Pancreatic adenocarcinoma, version 2.2017, NCCN Clinical Practice Guidelines in Oncology. J Natl Compr Canc Netw 2017;15:1028-1061.

18. Katz MH, Pisters PW, Evans DB, Sun CC, Lee JE, Fleming JB, et al. Borderline resectable pancreatic cancer: the importance of this emerging stage of disease. J Am Coll Surg 2008;206:833-846; discussion 846-848.

19. Ramacciato G, Nigri G, Petrucciani N, Pinna AD, Ravaioli M, Jovine E, et al. Pancreatectomy with mesenteric and portal vein resection for borderline resectable pancreatic cancer: multicenter study of 406 patients. Ann Surg Oncol 2016;23:2028-2037.

20. Ravikumar R, Sabin C, Abu Hilal M, Bramhall S, White S, Wigmore S, et al. Portal vein resection in borderline resectable pancreatic cancer: a United Kingdom multicenter study. J Am Coll Surg 2014;218:401-411.

21. Varty PP, Yamamoto H, Farges O, Belghiti J, Sauvanet A. Early retropancreatic dissection during pancreaticoduodenectomy. Am J Surg 2005;189:488-491.

22. Ironside N, Barreto SG, Loveday B, Shrikhande SV, Windsor JA, Pandanaboyana S. Meta-analysis of an artery-first approach versus standard pancreatoduodenectomy on perioperative outcomes and survival. Br J Surg 2018;105:628-636.

23. Horiguchi A, Ishihara S, Ito M, Nagata H, Shimizu T, Furusawa K, et al. Pancreatoduodenectomy in which dissection of the efferent arteries of the head of the pancreas is performed first. J Hepatobiliary Pancreat Surg 2007;14:575-578.

24. Inoue Y, Saiura A, Yoshioka R, Ono Y, Takahashi M, Arita J, et al. Pancreatoduodenectomy with systematic mesopancreas dissection using a supracolic anterior artery-first approach. Ann Surg 2015;262:1092-1101.

25. Daabiss M. American Society of Anaesthesiologists physical status classification. Indian J Anaesth 2011;55:111-115.

26. Negoi I, Hostiuc S, Runcanu A, Negoi RI, Beuran M. Superior mesenteric artery first approach versus standard pancreaticoduodenectomy: a systematic review and meta-analysis. Hepatobiliary Pancreat Dis Int 2017;16:127-138.

27. Cho A, Yamamoto H, Nagata M, Takiguchi N, Shimada H, Kainuma $\mathrm{O}$, et al. Comparison of laparoscopy-assisted and open pylorus-preserving pancreaticoduodenectomy for periampullary disease. Am J Surg 2009;198:445-449.

28. Song KB, Kim SC, Lee W, Hwang DW, Lee JH, Kwon J, et al. Laparoscopic pancreaticoduodenectomy for periampullary tumors: lessons learned from 500 consecutive patients in a single center. Surg Endosc 2020;34:1343-1352. 